\title{
Associations between cola consumption and bone mineral density in Korean adolescents and young adults: a cross-sectional study using data from the Korea National Health and Nutrition Examination Survey, 2008-2011
}

\author{
Yun-A $\operatorname{Kim}^{1}$ (1) and Jun-Hyun Yoo $^{2 *}$ \\ ${ }^{1}$ Department of Family Medicine, Daegu Catholic University School of Medicine, Daegu 42472, Republic of Korea \\ ${ }^{2}$ Department of Family Medicine, Sungkyunkwan University School of Medicine, Seoul 06351, Republic of Korea
}

(Received 29 May 2020 - Final revision received 14 October 2020 - Accepted 20 October 2020)

Journal of Nutritional Science (2020), vol. 9, e56, page 1 of 7

doi:10.1017/jns.2020.49

\section{Abstract}

The present study aimed to elucidate the relationship between cola consumption and bone mineral density (BMD) in Korean adolescents and young adults. We used data from the Korea National Health and Nutrition Examination Survey 2008-2011. A total of 2499 adolescents and young adults aged 12-25 years were included. The study participants were classified as cola drinkers and non-cola drinkers according to $24-\mathrm{h}$ dietary recall data. BMD was measured using dual X-ray absorptiometry. In the male population, whole body, whole femur and femoral neck BMD in cola drinkers were lower than that of noncola drinkers by $4 \%(95 \%$ CI $-0 \cdot 071,-0 \cdot 007), 5 \%(-0 \cdot 092,-0 \cdot 012)$ and $5 \%(-0 \cdot 090,-0 \cdot 001)$, respectively. In both sex groups, cola drinkers had less frequent milk consumption than non-cola drinkers. However, there were no significant differences in cola consumption according to calcium intake in both sexes. In conclusion, cola intake and BMD were inversely associated with Korean male adolescents and young adults. Considering the importance of peak bone mass attainment at adolescents and the increasing trend in carbonated beverage consumption in South Korea, further studies are needed to elucidate the causality between cola intake and lower BMD.

Key words: Peak bone mass: Bone mineral density: Cola: Adolescents: Young adults

\section{Introduction}

Low bone mineral density (BMD), whether osteopenia or osteoporosis, has become a worldwide interest as the population is ageing globally ${ }^{(1)}$. A progressive decline in BMD begins as peak bone mass (PBM) is reached between 20 and 30 years of age, which occurs initially independent of sex hormone levels ${ }^{(2)}$. Thus, both ageing and PBM attained during childhood and adolescence can be considered predictive factors of bone health at an older age ${ }^{(2,3)}$.

Bone mass gain is influenced by various factors, such as sex, ethnicity, hormonal status and lifestyle-related factors, including diet, physical activity, cigarette smoking and alcohol intake ${ }^{(3,4)}$. Several studies of children and young adults have also proposed that consumption of carbonated soft drinks is associated with low $\mathrm{BMD}^{(5,6)}$ and even increased risk of fractures $^{(7,8)}$. In one experimental study, a significant delay in alveolar bone formation was observed in cola consuming rats ${ }^{(9)}$. Furthermore, colas, but not other carbonated beverages, are related to low BMD in older women ${ }^{(10)}$ or increased upper limb fractures in children ${ }^{(11)}$. However, the results of the previous studies were different according to sex ${ }^{(5)}$, and the association between cola and BMD has been found mainly in women ${ }^{(7,10,12)}$. Displacement of beneficial nutrient-rich foods such as milk ${ }^{(5,13)}$, decreased physical

* Corresponding author: Jun-Hyun Yoo, email drjohn.yoo@samsung.com; dryoo@skku.edu

(C) The Author(s), 2020. Published by Cambridge University Press on behalf of The Nutrition Society. This is an Open Access article, distributed under the terms of the Creative Commons Attribution-NonCommercial-NoDerivatives licence (http://creativecommons.org/licenses/by-nc-nd/4.0/), which permits non-commercial re-use, distribution, and reproduction in any medium, provided the original work is unaltered and is properly cited. The written permission of Cambridge University Press must be obtained for commercial re-use or in order to create a derivative work. 
activity owing to watching television or computer while drinking colas ${ }^{(11)}$, and the presence of phosphoric acid and caffeine in colas that interfere with calcium absorption and excretion $^{(14,15)}$ have been suggested as possible mechanisms. However, a clear mechanism has not yet been proposed, and a set of contributing factors may exist ${ }^{(16)}$.

Most studies on the relationship between cola intake and BMD have been conducted with the Western populations, with very few studies involving Asian populations. Considering the clinical importance of PBM gain during childhood and adolescence and the increasing trend of consumption of carbonated soft drink in South Korea during this critical period for bone health ${ }^{(17,18)}$, examining whether cola is related to low BMD in the growing young population is worthwhile. Thus, we aimed to examine the associations between cola consumption and BMD in Korean adolescents and young adults.

\section{Methods}

\section{Data collection and study participants}

The present study was based on data acquired from the Korea National Health and Nutrition Examination Survey (KNHANES) from 2008 to 2011. The KNHANES is a nationwide and population-based cross-sectional health survey conducted periodically by the Korean Ministry of Health and Welfare. This survey consists of three distinctive surveys: health interviews; health examinations; nutrition. Data were collected via household interviews and by direct standardised physical examinations in specially equipped mobile examination centres. The survey methods have been described elsewhere $^{(19)}$.

BMD was initially measured in participants who were older than 19 years, but the target population was expanded to those who were older than 10 years since July 2009. A total of 20808 participants were assessed for the body composition using dual-energy X-ray absorptiometry (DXA) from 2008 to 2011. Among these participants, we limited our study population to adolescents and young adults aged 12-25 years, the age when BMD has been reported to increase and reach a plateau ${ }^{(2,20)}$. We excluded subjects who were diagnosed with asthma, liver cirrhosis, renal failure, thyroid disease or any kind of cancer that can affect BMD. Subjects who were being treated for osteoporosis were also excluded. To adjust for the sex hormone effect, premenarchal female participants were also excluded. Finally, a total of 1222 males and 1277 females aged 12-25 years were enrolled as the study population for analysis.

\section{BMD measurements}

BMD $\left(\mathrm{g} / \mathrm{cm}^{2}\right.$ ) and body composition were measured using DXA (Hologic Discovery, Hologic Inc., Bedford, MA, USA). As the posterior-anterior spine and whole body except the head are recommended skeletal sites for BMD measurements in paediatric populations ${ }^{(21)}$, we used BMD at the whole body which did not include the head, whole femur, femoral neck and lumbar spine for analysis.

\section{Dietary assessment}

The nutritional survey was conducted via a face-to-face interview with dietitians. The usual dietary habits were assessed using a 24-h dietary recall questionnaire, and the frequency of sixty-three food-item consumption was also investigated. The frequency of food consumption was based on dietary habits during the past 1 year. We assessed the daily intake amount of energy and other nutrients based on the calculated data using food frequency questionnaires presented in the KNHANES. We also evaluated whether calcium, phosphorus and energy intake were sufficient according to the dietary reference intake for Koreans ${ }^{(22)}$. Since the frequency of cola intake was not investigated in KNHANES, we estimated the level of cola consumption by summing the amount of cola intakes regardless of the brand, during a day using a 24-h dietary recall questionnaire. We also divided the study population into two groups based on their reports of cola consumption using a 24-h dietary recall data to examine the relationship between cola intake and BMD. Subjects who drank any kind of cola were categorised as cola drinkers. Subjects who did not reportedly drink any cola beverages were classified as non-cola drinkers. The amount of milk consumed daily was estimated using the same method. The frequency of food consumption was originally categorised into ten groups in the KNHANES: three times a day; twice a day; once a day; 4-6 times a week; 2-3 times a week; once a week; 2-3 times a month; once a month; 6-11 times a year; nearly nonexistent. We regrouped these ten groups into five categories to examine the distribution of coffee and milk consumption. 'Rarely' (represented subjects who reported consumption nearly none or 6-11 times a year), '1-3 times/month', '1-3 times/week', '4-7 times/week', ' $\geq$ twice a day'.

\section{Measurement of covariates}

Height and weight were measured using a standard method to the nearest $0.1 \mathrm{~cm}$ for height and $0.1 \mathrm{~kg}$ for weight. Waist circumference was measured at the midpoint between the lowest level of the ribs and iliac crest at the mid-axillary line. We used the calculated values of body mass index (BMI) presented in the original data. Lifestyle-related components were assessed using a self-administered questionnaire. Physical activity was categorised as insufficient exercise, which included no exercise and low-intensity exercise, and sufficient exercise, which included moderate-to-vigorous-intensity exercise. Low-intensity exercise was defined as walking for more than $5 \mathrm{~d} /$ week for more than $30 \mathrm{~min}$ at a time in the last week. Moderateto-vigorous-intensity exercise was defined as participating in moderate-intensity activities, such as swimming slowly or badminton, for more than $5 \mathrm{~d}$ /week for more than $30 \mathrm{~min}$ at a time or participating in vigorous-intensity activities, such as running, climbing or swimming fast for more than $3 \mathrm{~d}$ /week for more than $20 \mathrm{~min}$ at a time in the last week. Smoking status was divided into never smokers and ever smokers. Subjects who had smoked 100 cigarettes or more in their lifetime were categorised as ever smokers. Those who had never smoked or smoked less than 100 cigarettes in their lifetime were defined 
as never smokers. Alcohol consumption was assessed according to the frequency and amount of alcohol intake at a time. High-risk alcohol consumption was defined as drinking alcohol more than twice a week, with the average amount per drink of $\geq 7$ glasses for males, or $\geq 5$ glasses for females. We also assessed the serum $25(\mathrm{OH}) \mathrm{D}$ level.

\section{Statistical analyses}

All analyses were conducted using the SPSS software for Windows version 21.0 (SPSS Inc., Chicago, IL, USA). We used sampling weights based on the complex sample design of KNHANES in all analyses to obtain representatives of the Korean population. $\chi^{2}$ statistics and one-way analysis of variance were used to assess the difference between the baseline characteristics based on cola intake and sex. As the association between cola and BMD has been reported differently based on sex in previous studies, we examined the association stratified by sex first. Then, we divided the study population into two groups: cola drinkers and non-cola drinkers. To examine the association between calcium intake and cola consumption, we calculated the tertiles of daily calcium intake in both sexes. Multiple linear regression was used to examine the association between cola intake and BMD. Since BMD values were not normally distributed in this study population, they were log-transformed to approximate a normal distribution for the analyses. To examine the independent association between cola intake and BMD, we adjusted the potential influence of age, BMI, physical activity, smoking, alcohol consumption, daily calcium intake, daily phosphorus intake, total daily energy intake and serum $25(\mathrm{OH}) \mathrm{D}$ level. We additionally adjusted oestrogen exposure in the female population. Since the sex hormone level was not investigated in KNHANES, we estimated the degree of oestrogen exposure by subtracting the age of menarche from the present age. A $P$-value of less than $0 \cdot 05$ was considered statistically significant in all analyses.

\section{Ethical standards disclosure}

The present study was conducted according to the guidelines laid down in the Declaration of Helsinki and all procedures involving human subjects were approved by the Institutional Review Board at Sungkyunkwan University. All the participants in this survey signed an informed consent form, and legal guardian consent was obtained for participants aged under 14.

\section{Results}

\section{Characteristics of study participants}

Table 1 shows the baseline characteristics of the study population based on cola intake and sex. The numbers of noncola drinkers and cola drinkers were 1086 and 136 males and 1113 and 164 females. The differences in most clinical variables such as age, body composition parameters, lifestyle-related factors and serum 25(OH)D levels between cola drinkers and non-cola drinkers were not significant in both sexes, but differences in nutritional factors based on cola intake were significant. Daily energy, fat, carbohydrate and protein intake were significantly higher in male cola drinkers, and daily fat and protein intake were significantly higher in female cola drinkers than in their non-cola drinking female and male counterparts, respectively. However, calcium intake was significantly lower in cola drinkers than in non-cola drinkers in males, which was not observed in females. In addition, no significant differences were observed in the daily phosphorus intake based on cola intake status in either sex.

Other beverage consumption and calcium intake according to cola consumption

Table 2 shows the frequency of coffee and milk consumption based on cola intake and sex. Among males, 26.9\% cola drinkers $v \cdot 18.3 \%$ non-cola drinkers and among females, $35.8 \%$ cola drinkers $v .26 \cdot 0 \%$ non-cola drinkers drank coffee more than four times a week. There were was no statistically significant difference in coffee intake among the groups. In addition, among males, $35.5 \%$ cola drinkers $v .53 .8 \%$ non-cola drinkers and among females, $34.3 \%$ cola drinkers v. $43.7 \%$ non-cola drinkers drank milk more than four times a week; the differences were statistically significant. However, no significant difference in the daily amount of milk consumption was observed in either sex based on cola intake (data not shown).

Table 3 shows the daily amount of cola consumption according to daily calcium intake. The cut-off values of calcium intake in cola drinkers and non-cola drinkers were, respectively, 363.7 and $608.5 \mathrm{mg}$ in males and 280.2 and $499.1 \mathrm{mg}$ in females. Males who consumed less calcium among tertiles showed higher cola consumption on comparing the lowest tertile group $v$. the middle tertile group and the middle tertile group $v$. the highest tertile group. However, statistical significance was not observed among those associations. Females in the lowest tertile of calcium consumption had higher cola consumption than those in the middle and highest tertiles. However, the association between calcium intake and cola consumption was not statistically significant in female cola drinkers and non-drinkers.

\section{Relationship between cola intake and BMD}

Table 4 shows the association between cola intake and BMD at various sites. When adjusted for age and BMI only, the association between cola intake and BMD was not significant at all the measured sites. However, when lifestyle-related factors were adjusted more, the association between cola intake and BMD was inversely significant for the whole body in males. After adjusting the possible confounding factors regarding nutrients, male cola drinkers tended to have significantly lower BMD at the whole body, whole femur and femoral neck with 4, 5 and $5 \%$, respectively, compared with that of non-cola drinkers by applying the exponential function to the presented $\beta$. Lumbar spine BMD was also lower in male 
Table 1. Baseline characteristics of the study population ( $n$ 2499) stratified by cola intake and sex

\begin{tabular}{|c|c|c|c|c|c|c|c|c|c|c|}
\hline & \multicolumn{4}{|c|}{ Males } & \multirow[b]{3}{*}{$P$-value } & \multicolumn{5}{|c|}{ Females } \\
\hline & \multicolumn{2}{|c|}{$\begin{array}{c}\text { Non-cola } \\
\text { drinker }(n \text { 1086) }\end{array}$} & \multicolumn{2}{|c|}{ Cola drinker (n 136) } & & \multicolumn{2}{|c|}{$\begin{array}{c}\text { Non-cola } \\
\text { drinker }(n 1113)\end{array}$} & \multicolumn{2}{|c|}{ Cola drinker (n 164) } & \multirow[b]{2}{*}{$P$-value } \\
\hline & $\begin{array}{l}\text { Mean or } \\
\text { proportion }\end{array}$ & SE & $\begin{array}{c}\text { Mean or } \\
\text { proportion }\end{array}$ & SE & & $\begin{array}{l}\text { Mean or } \\
\text { proportion }\end{array}$ & SE & $\begin{array}{c}\text { Mean or } \\
\text { proportion }\end{array}$ & SE & \\
\hline Age (yr) & $18 \cdot 7$ & 0.2 & $19 \cdot 4$ & 0.4 & 0.09 & $18 \cdot 7$ & 0.2 & $18 \cdot 6$ & 0.4 & 0.74 \\
\hline Height (cm) & 171.9 & 0.3 & $172 \cdot 8$ & 0.7 & 0.29 & $160 \cdot 5$ & 0.2 & $160 \cdot 5$ & 0.5 & 0.97 \\
\hline Weight (kg) & $66 \cdot 0$ & 0.6 & 66.6 & 1.4 & 0.66 & $54 \cdot 2$ & 0.4 & $55 \cdot 2$ & $1 \cdot 2$ & 0.43 \\
\hline Waist circumference (cm) & $76 \cdot 1$ & 0.4 & $76 \cdot 6$ & 1.0 & 0.66 & $69 \cdot 4$ & 0.3 & $70 \cdot 4$ & 1.0 & 0.38 \\
\hline Body mass index $\left(\mathrm{kg} / \mathrm{m}^{2}\right)$ & $22 \cdot 2$ & 0.2 & $22 \cdot 3$ & 0.4 & 0.86 & $21 \cdot 0$ & 0.1 & 21.4 & 0.5 & 0.42 \\
\hline Lena body mass $(\mathrm{kg})$ & 50.9 & 0.4 & $51 \cdot 7$ & 0.9 & 0.43 & $36 \cdot 2$ & 0.2 & $36 \cdot 3$ & 0.5 & 0.92 \\
\hline \multicolumn{11}{|l|}{ Bone mineral density $\left(\mathrm{g} / \mathrm{cm}^{2}\right)$} \\
\hline Whole body & 0.990 & 0.005 & 0.980 & 0.010 & 0.46 & 0.890 & 0.004 & 0.890 & 0.010 & 0.64 \\
\hline Whole femur & 0.979 & 0.005 & 0.975 & 0.015 & 0.82 & 0.879 & 0.004 & 0.889 & 0.001 & 0.39 \\
\hline Femoral neck & 0.885 & 0.005 & 0.880 & 0.016 & 0.75 & 0.769 & 0.004 & 0.789 & 0.010 & 0.13 \\
\hline Lumbar spine & 0.926 & 0.006 & 0.931 & 0.014 & 0.74 & 0.921 & 0.005 & 0.947 & 0.013 & 0.07 \\
\hline Physical activity (\%) & & & & & 0.77 & & & & & 0.39 \\
\hline Insufficient exercise & $55 \cdot 2$ & $3 \cdot 1$ & $58 \cdot 1$ & $9 \cdot 6$ & & $69 \cdot 1$ & 3.0 & $76 \cdot 2$ & $7 \cdot 2$ & \\
\hline Sufficient exercise & $44 \cdot 8$ & $3 \cdot 1$ & 41.9 & $9 \cdot 6$ & & 30.9 & 3.0 & $23 \cdot 8$ & $7 \cdot 2$ & \\
\hline Ever smoker (\%) & 39.6 & 1.9 & $36 \cdot 6$ & $6 \cdot 0$ & 0.64 & $12 \cdot 5$ & $1 \cdot 2$ & $16 \cdot 6$ & $3 \cdot 2$ & 0.24 \\
\hline High-risk alcohol consumption (\%) & $9 \cdot 8$ & $1 \cdot 3$ & $9 \cdot 3$ & 3.0 & 0.88 & 2.4 & 0.5 & 1.7 & $1 \cdot 1$ & 0.61 \\
\hline Serum 25(OH)D (ng/ml) & 16.4 & 0.2 & $16 \cdot 3$ & 0.7 & 0.94 & $15 \cdot 0$ & 0.3 & 14.4 & 0.5 & 0.35 \\
\hline Daily energy intake (kcal) & $2356 \cdot 4$ & $48 \cdot 1$ & 2774.8 & $122 \cdot 8$ & $<.001$ & $1767 \cdot 4$ & $29 \cdot 2$ & $1942 \cdot 8$ & $38 \cdot 2$ & 0.05 \\
\hline Daily fat intake $(g)$ & 62.8 & 1.9 & 78.8 & $5 \cdot 8$ & 0.01 & $46 \cdot 6$ & $1 \cdot 3$ & 55.8 & $3 \cdot 6$ & 0.02 \\
\hline Daily carbohydrate intake (g) & $344 \cdot 2$ & $6 \cdot 1$ & $379 \cdot 6$ & $14 \cdot 8$ & 0.03 & 271.9 & 4.5 & $276 \cdot 3$ & $10 \cdot 1$ & 0.69 \\
\hline Daily protein intake (g) & $86 \cdot 3$ & $2 \cdot 2$ & $100 \cdot 7$ & 5.6 & 0.02 & $62 \cdot 2$ & $1 \cdot 2$ & 70.7 & $3 \cdot 8$ & 0.05 \\
\hline Daily calcium intake (mg) & $557 \cdot 0$ & $16 \cdot 3$ & 478.6 & $25 \cdot 3$ & 0.01 & 431.4 & $10 \cdot 6$ & $393 \cdot 7$ & $19 \cdot 0$ & 0.09 \\
\hline Daily phosphorus intake (mg) & 1339.9 & $28 \cdot 1$ & $1344 \cdot 8$ & $61 \cdot 2$ & 0.94 & 987.5 & $17 \cdot 0$ & $986 \cdot 3$ & $45 \cdot 3$ & 0.98 \\
\hline
\end{tabular}

SE, standard error.

cola drinkers than in non-cola drinkers. However, statistical significance was not observed in this inverse association. In females, whole body BMD was inversely associated with cola intake when all the possible confounding factors were adjusted for, although no statistical significance was observed. In addition, no significant association was observed at other skeletal sites in females. When all confounding factors were adjusted as for model 3 except phosphorus, the coefficients were not found to be significantly different in all measured skeletal sites (data not shown).

\section{Discussion}

In the present study, we observed that male cola drinkers had lower BMD at the whole body, whole femur and femoral neck than non-cola drinkers after adjusting for possible confounding factors. We also found that cola drinkers had less frequent milk consumption than non-cola drinkers, but the average cola consumption was not statistically different according to calcium intake in both sexes.

In an observational study of Northern Irish adolescents aged 12 and 15 years, a significant inverse association between

Table 2. Beverage consumption in the study population ( $n$ 2499) stratified by cola intake and sex

\begin{tabular}{|c|c|c|c|c|c|c|c|c|c|c|}
\hline & \multicolumn{4}{|c|}{ Males } & \multirow[b]{3}{*}{$P$-value } & \multicolumn{4}{|c|}{ Females } & \multirow[b]{3}{*}{$P$-value } \\
\hline & \multicolumn{2}{|c|}{$\begin{array}{c}\text { Non-cola } \\
\text { drinker }(n \text { 1086) }\end{array}$} & \multicolumn{2}{|c|}{$\begin{array}{l}\text { Cola drinker } \\
\quad(n \text { 136) }\end{array}$} & & \multicolumn{2}{|c|}{$\begin{array}{c}\text { Non-cola } \\
\text { drinker }(n 1113)\end{array}$} & \multicolumn{2}{|c|}{$\begin{array}{l}\text { Cola drinker } \\
\quad(n \text { 164) }\end{array}$} & \\
\hline & $\%$ & SE & $\%$ & SE & & $\%$ & SE & $\%$ & SE & \\
\hline Coffee consumption & & & & & 0.25 & & & & & 0.28 \\
\hline Rarely & 42.4 & $2 \cdot 0$ & $31 \cdot 8$ & $5 \cdot 6$ & & 41.5 & $2 \cdot 0$ & 34.7 & 5.0 & \\
\hline $1-3$ times/month & $17 \cdot 0$ & 1.6 & $18 \cdot 6$ & $4 \cdot 1$ & & 13.8 & $1 \cdot 2$ & $11 \cdot 8$ & 3.0 & \\
\hline 1-3 times/week & $22 \cdot 3$ & $1 \cdot 7$ & $22 \cdot 7$ & 4.5 & & $18 \cdot 7$ & 1.5 & $17 \cdot 7$ & $3 \cdot 3$ & \\
\hline 4-7 times/week & $14 \cdot 0$ & 1.5 & $19 \cdot 6$ & $4 \cdot 2$ & & $17 \cdot 6$ & 1.5 & 26.5 & $5 \cdot 2$ & \\
\hline$\geq$ twice a day & $4 \cdot 3$ & 0.9 & $7 \cdot 3$ & $2 \cdot 7$ & & 8.4 & $1 \cdot 1$ & $9 \cdot 3$ & $2 \cdot 8$ & \\
\hline Milk consumption & & & & & $<0.001$ & & & & & 0.02 \\
\hline Rarely & $5 \cdot 0$ & 0.9 & $13 \cdot 0$ & 3.7 & & 13.8 & 1.5 & 9.4 & 2.9 & \\
\hline $1-3$ times/month & $10 \cdot 1$ & 1.3 & $6 \cdot 0$ & $2 \cdot 0$ & & $10 \cdot 8$ & 1.2 & 9.5 & $2 \cdot 4$ & \\
\hline 1-3 times/week & $31 \cdot 1$ & $2 \cdot 1$ & 45.5 & $5 \cdot 8$ & & 31.8 & 1.8 & $46 \cdot 9$ & 4.1 & \\
\hline 4-7 times/week & 46.0 & $2 \cdot 0$ & $28 \cdot 2$ & $5 \cdot 0$ & & 39.1 & 1.9 & $32 \cdot 4$ & 4.1 & \\
\hline$\geq$ twice a day & 7.8 & 1.0 & $7 \cdot 3$ & $2 \cdot 6$ & & 4.6 & 0.7 & 1.9 & $1 \cdot 2$ & \\
\hline
\end{tabular}

$\mathrm{SE}$, standard error. 
Table 3. Daily amount of cola consumption according to calcium intake in each sex

\begin{tabular}{lrrr}
\hline & \multicolumn{2}{c}{ Daily cola } \\
& consumption $(\mathrm{g})$ & \\
\cline { 2 - 3 } & Mean & SE & $P$ for trend \\
\hline Males $(n$ 1222) & & & 0.62 \\
T1 & 54.8 & 9.6 & \\
T2 & 52.9 & 10.6 & \\
T3 & 42.5 & 9.3 & \\
Females $(n$ 1277) & & & 0.56 \\
T1 & 46.6 & 7.0 & \\
T2 & 36.5 & 5.9 & \\
T3 & 40.4 & 10.5 & \\
\hline
\end{tabular}

SE, standard error.

${ }^{a}$ T1, lowest tertile group; T2, middle tertile group; T3, highest tertile group. Cut-off values were 363.7 and $608.5 \mathrm{mg} / \mathrm{d}$ in males and 280.2 and $499.1 \mathrm{mg} / \mathrm{d}$ in females, respectively.

total carbonated soft drink intake and BMD at the dominant heel was observed in girls. However, no consistent associations were found in boys ${ }^{(5)}$. Similarly, a significant association between cola intake and increased risk of fracture was also reported in girls $^{(7)}$. In addition, a significant association between cola intake and lower hip BMD was reported in adults, which was confined to female participants ${ }^{(10)}$. In agreement with these previous studies, we observed that BMD was inversely associated with cola intake. We also observed various inverse associations between cola intake and BMD depending on the measured skeletal sites, which was consistent with previous findings ${ }^{(5,10)}$. Considering that each bone is composed of different materials and the trabecular bone is more metabolically active than cortical bone ${ }^{(23)}$, our findings might be influenced by various susceptibilities to nutritional influences based on the type of bone.

However, contrary to previous results, only male participants showed a significant inverse association between cola intake and BMD in the present study. When comparing the average amount of cola consumption according to sex in cola drinkers, female participants were found to consume $263.4 \pm 20.7 \mathrm{~g} / \mathrm{d}$ and male participants were found to consume $314.5 \pm 24.9 \mathrm{~g} / \mathrm{d}$ (data not shown). Since female cola drinkers consumed relatively small amounts of cola, the effects of cola on BMD may have been weakened. In addition, while the average daily calcium intake was $800-1000 \mathrm{mg} / \mathrm{d}$ in the previous studies on Western populations, in the present study, it was $544.5 \pm 13.7 \mathrm{mg} / \mathrm{d}$ in males and $425.5 \pm 9.6 \mathrm{mg} / \mathrm{d}$ in females, respectively (data not shown). Approximately $75 \%$ of Korean adolescents report inadequate calcium intake, and its prevalence is increasing with age in children and adolescents ${ }^{(24)}$. The aforementioned previous studies examined subjects with sufficient calcium intake; therefore, the results of studies involving subjects with inadequate calcium intake may show different results. In addition, due to the lack of information in the original data, we were unable to assess hormonal interactions in the male participants. Although we adjusted for oestrogen exposure in females, the absence of full assessment of pubertal status in both sexes might weaken the associations observed in the present study. However, considering BMD increases in proportion to age ${ }^{(25)}$ and reaches a plateau after completion of growth at about 16-18 years of age in females and 17-20 years of age in males ${ }^{(26)}$, the effect of pubertal status on BMD might have been adjusted to some degree by adjustment for chronological age. Additional research is needed to examine the different associations between cola intake and BMD based on sex and pubertal status.

We found that cola intake was associated with low BMD. However, we could not investigate how it is directly linked to low BMD. Some hypotheses have been proposed to explain the relationship between cola intake and BMD. Phosphoric acid and caffeine in cola beverages have been suggested to have deleterious effects on the bone ${ }^{(12,14,15,27)}$. However, no attenuated inverse association between cola intake and BMD was reported after adjusting for the calcium to phosphorus ratio or total caffeine intake ${ }^{(10)}$, which suggests that other components of cola might play a role in this regard. In addition, since there were no significant associations between total intake of carbonated beverages or carbonated beverages except cola and BMD (data not shown), it can be said that certain components only contained in cola affect the bone.

Table 4. Multivariate-adjusted ${ }^{\mathrm{a}}$ association between cola intake and bone mineral density ${ }^{\mathrm{b}}$ at various sites

\begin{tabular}{|c|c|c|c|c|c|c|c|c|}
\hline & \multicolumn{2}{|c|}{ Whole body } & \multicolumn{2}{|c|}{ Whole femur } & \multicolumn{2}{|c|}{ Femoral neck } & \multicolumn{2}{|c|}{ Lumbar spine } \\
\hline & $\beta$ & $95 \% \mathrm{Cl}$ & $\beta$ & $95 \% \mathrm{Cl}$ & $\beta$ & $95 \% \mathrm{Cl}$ & $\beta$ & $95 \% \mathrm{Cl}$ \\
\hline \multicolumn{9}{|c|}{ Males ( $n$ 1222) } \\
\hline Model 1 & -0.011 & $-0.031,0.008$ & -0.013 & $-0.043,0.016$ & -0.015 & $-0.048,0.018$ & -0.007 & $-0.035,0.020$ \\
\hline Model 2 & -0.032 & $-0.061,-0.002$ & -0.029 & $-0.072,0.014$ & -0.026 & $-0.074,0.023$ & -0.015 & $-0.058,0.028$ \\
\hline Model 3 & -0.039 & $-0.071,-0.007$ & -0.052 & $-0.092,-0.012$ & -0.046 & $-0.090,-0.001$ & -0.027 & $-0.071,0.017$ \\
\hline \multicolumn{9}{|c|}{ Females ( $n$ 1277) } \\
\hline Model 1 & 0.001 & $-0.013,0.016$ & 0.004 & $-0.017,0.025$ & 0.018 & $-0.008,0.044$ & 0.020 & $-0.002,0.037$ \\
\hline Model 2 & 0.006 & $-0.020,0.032$ & 0.000 & $-0.034,0.035$ & 0.009 & $-0.027,0.046$ & 0.025 & $-0.005,0.055$ \\
\hline Model 3 & -0.008 & $-0.043,0.028$ & 0.019 & $-0.018,0.056$ & 0.021 & $-0.033,0.076$ & 0.023 & $-0.011,0.056$ \\
\hline
\end{tabular}

$\mathrm{Cl}$, confidence interval.

${ }^{a}$ Model 1: adjusted for age and body mass index.Model 2: adjusted for age, body mass index, physical activity, smoking and alcohol consumption.Model 3: adjusted for age, body mass index, physical activity, smoking, alcohol consumption, daily calcium intake, daily phosphorus intake, daily protein intake, serum $25(\mathrm{OH}) \mathrm{D}$ level and total daily energy intake. Oestrogen exposure was additionally adjusted for females.

${ }^{b}$ Log-BMD was used for multiple linear regression analysis. 
Decreased milk consumption was found to be related to increased carbonated beverage intake in Irish adolescents ${ }^{(5)}$. Similar to these previous findings, we found a significant difference in milk consumption between cola drinkers and noncola drinkers. However, there was no difference in the daily amount of milk consumption and phosphorus intake between cola drinkers and non-cola drinkers in either sex (data not shown). Furthermore, the inverse associations between cola and BMD did not differ with adjustment for phosphorus intake. Regarding the effect of phosphoric acid from cola and other foods, we could not determine the source of phosphorus since the KNHANES recorded only the total daily amount of phosphorus intake and not the sources. Based on our analysis after adjusting for daily total phosphorus intake, the inverse association observed between cola consumption and BMD did not seem to originate from phosphoric acid.

In the present study, calcium consumption was significantly lower in cola drinkers, which is consistent with previous findings ${ }^{(5,10)}$. Although the statistical significance was not observed in females $(P=0.09)$, cola drinkers of both sexes consumed less calcium than non-cola drinkers. However, the average amount of cola consumption was not different among groups based on daily calcium intake and sex. Further studies are needed to elucidate whether specific components in cola play a role in lowering BMD and whether certain dietary habits are linked to cola consumption.

In the present study, the average amount of cola consumption was $35 \mathrm{~g} / \mathrm{d}$ in female participants, and $40 \mathrm{~g} / \mathrm{d}$ in male participants (data not shown), which was lower than the $230 \mathrm{~g} / \mathrm{d}$ in girls and $320 \mathrm{~g} / \mathrm{d}$ in boys, as reported in the Irish study on 12 - and 15 -year-olds ${ }^{(5)}$. The relatively low amount of cola consumption found in the present study might have attenuated the relationship between cola and BMD. However, the consumption of carbonated soft drinks has been steadily increasing in South Korea, reaching 29.6 l of annual consumption per capita in $2014^{(17)}$. This indicates that the Korean population consumes an average of approximately 0.4 cup of carbonated soft drink per day. In addition, the average frequency of carbonated soft drink consumption in South Korean adolescents and young adults is $1-3$ times a week with a tendency to decrease with age, suggesting that adolescents are the main consumers of carbonated beverages in South Korea ${ }^{(28)}$. In a survey of males and females in their teens to 40s, cola was ranked the primarily purchased soda among all carbonated soft drinks $(42 \%)$, followed by cider, fruit-flavoured soda and other sodas ${ }^{(17)}$. Moreover, Korean cola consumption per capita exceeded the worldwide consumption in 2012, according to a cola company report ${ }^{(29)}$. Considering the trends of increase in the consumption of carbonated soft drinks including colas in South Korea, the inverse association between cola intake and BMD observed in the present study may be a matter of concern.

To the best of our knowledge, this is the first study to investigate the association between cola intake and BMD in South Korea. However, the causal relationship between cola intake and BMD could not be assessed because of the cross-sectional nature of the study. In addition, since we used self-reported questionnaires and 24-h dietary recall data, we were unable to investigate and quantify the total lifetime cola consumption. Moreover, the average amount of beverage consumption, including cola, in the present study, might not represent the usual consumption of the study participants. People who occasionally drink cola might have not had cola on the day of the examination, which would attenuate the association between cola and BMD found in the present study. In addition, we did not classify the colas according to the degree of caffeine content, which made it impossible to assess the effect of caffeine on BMD. Moreover, the hidden confounding effects of certain medications, such as corticosteroids, or certain diseases that were not excluded, such as hypogonadism or parathyroid disorders, might have affected the results. We also could not assess parathyroid hormone (PTH) levels in our study population since PTH levels were only assessed among those over 50 years of age in the KNHANES. With regard to statistical methods, we examined an extensive series of covariates affecting bone health to minimise the effects of possible confounders. However, this might produce a misleadingly high explanatory power of the regression model since several covariates were adjusted for in the analysis. Despite these limitations, since the present study was based on nationwide and population-based data in South Korea, our findings can be applied to the general population of South Korean adolescents and young adults.

In conclusion, the present study demonstrates that cola intake was inversely associated with BMD in Korean male adolescents and young adults. Considering the increase in the trend of consumption in carbonated beverages in South Korea, further studies are required to elucidate the causality between carbonated beverages and lower BMD and whether the mechanism underlying the effect of carbonated beverages on bone density is mediated by sex.

\section{Acknowledgements}

We thank professor Yun Mi Song and professor Jungkwon Lee in the Department of Family Medicine, Sungkyunkwan University School of Medicine for their comments that greatly improved the manuscript.

This research received no specific grant from any funding agency, commercial or not-for-profit sectors.

J.H.Y. developed the research questions and made substantial contributions to the study design. Y.A.K. performed the main analyses and contributed to manuscript writing and submission. All authors were involved in the review and revision of the manuscript and approved the final manuscript.

The authors declare that they have no conflicts of interest.

\section{References}

1. Friedman SM \& Mendelson DA (2014) Epidemiology of fragility fractures. Clin Geriatr Med 30, 175-181.

2. Hendrickx G, Boudin E \& Van Hul W (2015) A look behind the scenes: the risk and pathogenesis of primary osteoporosis. Nat Rev Rheumatol 11, 462-474.

3. Rizzoli R, Bianchi ML, Garabedian M, et al. (2010) Maximizing bone mineral mass gain during growth for the prevention of fractures in the adolescents and the elderly. Bone 46, 294-305. 
4. Curtis E, Litwic A, Cooper C, et al. (2015) Determinants of muscle and bone aging. J Cell Physiol 230, 2618-2625.

5. McGartland C, Robson PJ, Murray L, et al. (2003) Carbonated soft drink consumption and bone mineral density in adolescence: the Northern Ireland young hearts project. J Bone Miner Res 18, 1563-1569.

6. Alghadir AH, Gabr SA \& Al-Eisa E (2015) Physical activity and lifestyle effects on bone mineral density among young adults: sociodemographic and biochemical analysis. J Phys Ther Sci 27, 2261-2270.

7. Wyshak G (2000) Teenaged girls, carbonated beverage consumption, and bone fractures. Arch Pediatr Adolesc Med 154, 610-613.

8. Manias K, McCabe D \& Bishop N (2006) Fractures and recurrent fractures in children: varying effects of environmental factors as well as bone size and mass. Bone 39, 652-657.

9. Teofilo JM, Leonel DV \& Lamano T (2010) Cola beverage consumption delays alveolar bone healing: a histometric study in rats. Braz Oral Res 24, 177-181.

10. Tucker KL, Morita K, Qiao N, et al. (2006) Colas, but not other carbonated beverages, are associated with low bone mineral density in older women: the Framingham Osteoporosis Study. Am J Clin Nutr 84, 936-942.

11. Ma D \& Jones G (2004) Soft drink and milk consumption, physical activity, bone mass, and upper limb fractures in children: a population-based case-control study. Calcif Tissue Int 75, 286-291.

12. Wyshak G \& Frisch RE (1994) Carbonated beverages, dietary calcium, the dietary calcium/phosphorus ratio, and bone fractures in girls and boys. J Adolesc Health 15, 210-215.

13. Kristensen M, Jensen M, Kudsk J, et al. (2005) Short-term effects on bone turnover of replacing milk with cola beverages: a 10-day interventional study in young men. Osteoporos Int 16, 1803-1808.

14. Amato D, Maravilla A, Montoya C, et al. (1998) Acute effects of soft drink intake on calcium and phosphate metabolism in immature and adult rats. Rev Invest Clin 50, 185-189.

15. Rapuri PB, Gallagher JC, Kinyamu HK, et al. (2001) Caffeine intake increases the rate of bone loss in elderly women and interacts with vitamin D receptor genotypes. Am J Clin Nutr 74, 694-700.

16. Fung TT, Arasaratnam MH, Grodstein F, et al. (2014) Soda consumption and risk of hip fractures in postmenopausal women in the Nurses' Health Study. Am J Clin Nutr 100, 953-958.
17. Korea Agro-Fisheries \& Food Trade Corporation, et al. (2015) Detail status of processed foods.

18. Han E, Kim TH \& Powell LM (2013) Beverage consumption and individual-level associations in South Korea. BMC Public Health 13, 195.

19. Lim JS, Kim KM, Rhee Y, et al. (2012) Gender-dependent skeletal effects of vitamin $\mathrm{D}$ deficiency in a younger generation. $J$ Clin Endocrinol Metab 97, 1995-2004.

20. Kang MJ, Hong HS, Chung SJ, et al. (2016) Body composition and bone density reference data for Korean children, adolescents, and young adults according to age and sex: results of the 2009-2010 Korean National Health and Nutrition Examination Survey (KNHANES). J Bone Miner Metab 34, 429-439.

21. Crabtree NJ, Arabi A, Bachrach LK, et al. (2014) Dual-energy X-ray absorptiometry interpretation and reporting in children and adolescents: the revised 2013 ISCD pediatric official positions. J Clin Densitom 17, 225-242.

22. Ministry of Health \& Welfare, et al. (2015) Dietary reference intakes for Koreans 2015.

23. Rauch F \& Schoenau E (2001) Changes in bone density during childhood and adolescence: an approach based on bone's biological organization. J Bone Miner Res 16, 597-604.

24. Im JG, Kim SH, Lee GY, et al. (2014) Inadequate calcium intake is highly prevalent in Korean children and adolescents: the Korea National Health and Nutrition Examination Survey (KNHANES) 2007-2010. Public Health Nutr 17, 2489-2495.

25. Moretto de Oliveria MR, Cristiane da Silva C, Kurokawa CS, et al. (2011) Bone mineral density in healthy female adolescents according to age, bone age and pubertal breast stage. Open Orthop J 5, 324-330.

26. Davies JH, Evans BA \& Gregory JW (2005) Bone mass acquisition in healthy children. Arch Dis Child 90, 373-378.

27. Fernando GR, Martha RM \& Evangelina R (1999) Consumption of soft drinks with phosphoric acid as a risk factor for the development of hypocalcemia in postmenopausal women. J Clin Epidemiol 52, 1007-1010.

28. Korea Health Industry Development Institute (2006) Youth health and carbonated drink.

29. The Coca-Cola Company (2012) Per capita consumption of company beverage products. 